\title{
Research of City Engineering Speech Blind Separation Algorithms Based on Independent Vector Analysis
}

\author{
Zhuo Yang ${ }^{1, a}$, Chun-ming $\mathrm{Li}^{2, \mathrm{~b}}$ \\ ${ }^{1}$ Electromechanics Department, Zhengzhou Tourism College, Zhengzhou 450009, Peoples R \\ China \\ ${ }^{2}$ Electromechanics Department, Zhengzhou Tourism College, Zhengzhou 450009, Peoples R \\ China \\ aemail: yangzhuo20112012@163.com, bemail: chunmingli2012@163.com
}

Keywords: Blind Separation; Independent Vector; Convolutive Mixture; Voice Signal

\begin{abstract}
Aiming at the rapid expansion of urbanization, This paper proposed a new independent vector analysis model, it is an extension of ICA for independent multivariate speech where the sources are mixed component-wise. Formulation that can solve the frequency-domain blind source separation problem, which is very useful in directly applying Newton's method to a contrast function of complex-valued variables. Fast fixed-point independent vector analysis blind source separation algorithms are derived .The real speech signal are used to demonstrate the good converge and accuracy of the algorithms by simulating.
\end{abstract}

\section{Introduction}

The rapid expansion of urbanization is increasingly putting impacts on speech blind separation, and the traditional methods of speech blind separation have exposed their limitations under new circumstances, with the application of fast fixed-point independent vector analysis into it, the unban speech blind separation is instantly developing unprecedentedly[1]. Independent component analysis is a famous and has been widely used in the "blind source separation" of the algorithm. It assumes that the original signal statistical independence, through the output signal the greatest degree of statistical independence, and separate source signal. However, in most of the actual environment, speech signal through the media time delay and reverberation effect will happen, so the speech signal problems based on blind source separation is mixed convolution, and in the frequency domain separation method of the values of the complex signal often also[2].

This makes simple ICA model has no longer apply and need to expand and the formation of ICA model delay and convolution mixed model. And at present most speech signal processing algorithm is blind is mostly by the nonstationary blind and non-ferrous signal processing algorithm to blind separation [3], separation results not ideal.

This paper puts forward designated independent vector analysis of fast separation algorithm, the time delay and convolution mixture model from the time domain is mapped to the frequency domain and time domain convolution the mixed problem into frequency domain each frequency mixing the instantaneous separated, the whole frequency domain into $F$ a frequency of each frequency application instantaneous linear separation method separation [4] [5]. And the algorithm by increasing the frequency of the signal source between independent constraints to better solves the blind source separation of frequency domain in order not to determine problem [6].

\section{Independent Vector Analysis (IVA)}

The traditional methods of speech blind separation have exposed their limitations under new circumstances, with the application of fast fixed-point independent vector analysis into it, a new independent vector analysis model is proposed. In the time $t$ domain frequency domain with the corresponding $n$ said time, $n$ corresponding to each short-time Fourier transform [7]. Therefore, independent vector analysis can be expressed as: 


$$
\begin{aligned}
& X^{f}[n]=A^{f} S^{f}[n] \\
& Y^{f}[n]=W^{f} \cdot X^{f}[n]
\end{aligned}
$$

Aiming at the independent vector analysis, in Eq. (1) and Eq. (2)

$$
\begin{aligned}
& Y^{f}[n]=\left[y_{1}{ }^{f}[n], y_{2}{ }^{f}[n], \ldots y_{n}{ }^{f}[n]\right]^{T} \\
& X^{f}[n]=\left[{x_{1}}^{f}[n], x_{2}{ }^{f}[n], \ldots x_{n}{ }^{f}[n]\right]^{T} \\
& S^{f}[n]=\left[s_{1}{ }^{f}[n],{s_{2}}^{f}[n], \ldots s_{n}{ }^{f}[n]\right]^{T}
\end{aligned}
$$

Among them, $f$ is one point for each frequency, $F$ for the number of frequency. In (1) through the different layers of the source signal is independent, so can form vector. $S_{1}$ and $S_{2}$ are multiple source signals, $X_{1}$ and $X_{2}$ are observation vector.

Obviously, in each layer in multiple source signals still mixes and forms by components form ICA mixed model. Independent vector analysis and multiple of independent component analysis is different [8]: IVA have multiple standards ICA levels constitute [9] [10] [11], each ICA layer in form component mixed together, and independent source signal keep not be mixed, so they can form a vector; And diverse ICA (MICA) only have a mixing layer, and independent source signal was mixed, so he can't be divided into several ICA layer. And this paper described the basic algorithm to solve the IVA ICA algorithm order in a sequence of uncertainty. Rapid designated independent vector analysis suitable for frequency domain without noise convolution blind source separation [12].

\section{Blind Separation Based on Fast Fixed-point Independent Vector Algorithm}

Aiming at the rapid expansion of urbanization, this paper proposed a new independent vector analysis model; it is an extension of ICA for independent multivariate speech where the sources are mixed component-wise. Because of the complex variable control function is hard up, so we put RenYiFu variable $Z$ transformation for $z=u+j v$, then carries on the analysis[13][14], we'll put $z$ real part of the imaginary part and in real field is optimized. Usually, the complex variable show source as the center of the circle symmetry, so that the source for vector round:

$$
E\left[S S^{T}\right]=\mathrm{O}
$$

In the new independent vector analysis model, set a voice for the frequency of the signal components is $\hat{f}_{s_{i}}$, through the observation of our attention to natural signals such as voice signal has round invariance characteristic, therefore the symmetry index distribution into round

$$
\hat{f}_{s_{i}}(z) \propto \frac{e-\sqrt{(4 / d)}\|z\|_{2}}{\|z\|_{2}^{d-1}}
$$

The ICA algorithm of speech blind separation has two keys, one is the choice of control function, and the other one is to control function optimization. Most part of the ICA algorithm USES the steepest descent method, natural gradient method or rapid fixed-point, Newton iterative method to optimize control function. Newton method is adopted in this paper to update the rules, in this convergence fast and good robustness. This article applies after gradient and complex Hessian matrix, and through the linear transformation of the relationship between the one-to-one up.

$$
\begin{aligned}
& W=\left[W_{1}, W_{2} \cdots\right]^{T} \\
& =u+j v=\left[u_{1}, u_{2} \cdots\right]^{T}+j\left[v_{1}, v_{2} \cdots\right]^{T} \\
& z=\left[u_{1}, v_{1}, u_{2}, v_{2} \cdots\right]^{T} \\
& W_{D}=\left[w_{1}, w_{1}{ }^{*}, w_{2}, w_{2}{ }^{*} \cdots\right]^{T} \\
& g(w)=g_{D}\left(w, w^{*}\right)=h(u, v)
\end{aligned}
$$


Among them $u$ and $v$ for real vector, and $h(u, v)$ about $z=o$ two Taylor polynomial equivalents to the expression of the complex Taylor polynomial:

$$
\begin{aligned}
& h(o, o)+\frac{\partial h(o, o)}{\partial Z^{T}} Z+\frac{1}{2} Z^{T} \frac{\partial^{2} h(o, o)}{\partial Z \cdot \partial Z^{T}} Z \\
= & g_{D}(o, o)+\frac{\partial g_{D}(o, o)}{\partial W_{D}{ }^{T}} W_{D}+\frac{1}{2} W_{D}{ }^{T} \frac{\partial^{2} g_{D}(o, o)}{\partial W_{D} \cdot \partial W_{D}{ }^{T}} W_{D}
\end{aligned}
$$

Do transform $W_{D}=\left[w ; w^{*}\right]$ and into (12) type

$$
\begin{aligned}
g(W) & \approx g\left(w_{o}\right)+\frac{\partial g\left(w_{o}\right)}{\partial w^{T}}\left(w-w_{o}\right)+\frac{\partial g\left(w_{o}\right)}{\partial w^{H}}\left(w-w_{o}\right)^{*}+\frac{1}{2}\left(w-w_{o}\right)^{T} \frac{\partial^{2} g\left(w_{o}\right)}{\partial w \cdot \partial w^{T}}\left(w-w_{o}\right) \\
& +\frac{1}{2}\left(w-w_{o}\right)^{H} \frac{\partial^{2} g\left(w_{o}\right)}{\partial w^{*} \cdot \partial w^{H}}\left(w-w_{o}\right)^{*}+\left(w-w_{o}\right)^{H} \frac{\partial^{2} g\left(w_{o}\right)}{\partial w^{*} \cdot \partial w^{T}}\left(w-w_{o}\right)
\end{aligned}
$$

In the type (13) Taylor expansion from $w=o$ point has become $w=w_{o}$.In the application of Newton method, $w$ the substituted for $w_{i}{ }^{f}$, get Newton iterative algorithm for

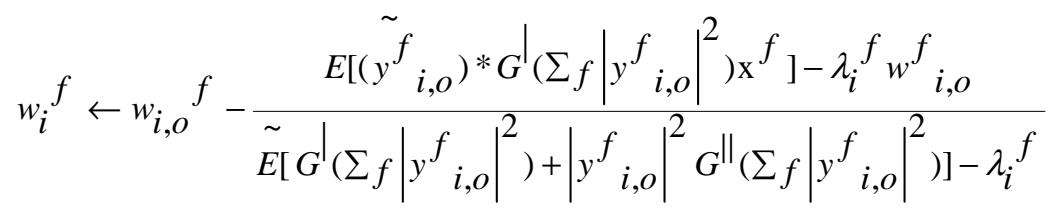

\section{IVA Analysis of Voice Signal}

We put the rapid fixed-point IVA algorithm used in speech signal separation. Two speech signal from the two girls speech by two about $3 \mathrm{~cm}$ microphones captured and signal reverberation time ten seconds, 1024 DFT sampling points. Matlab randomly generated by the mixed array for $\mathrm{A}=\left[\mathrm{a}_{11}\right.$, $\mathrm{a}_{21}, \mathrm{a}_{12}, \mathrm{a}_{22}$.

For with noise random mixture of aliasing first speech signal denoising, get a "pure" no chirp voice signal to noise of speech signal after mixing of the application of the designated IVA separation algorithm is fast, The results are as follows:

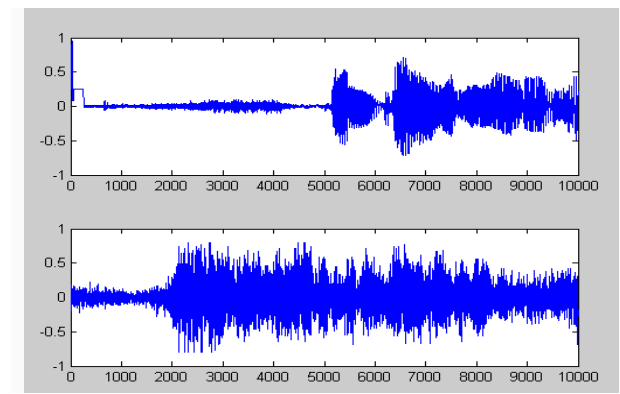

(a) Original sound signal

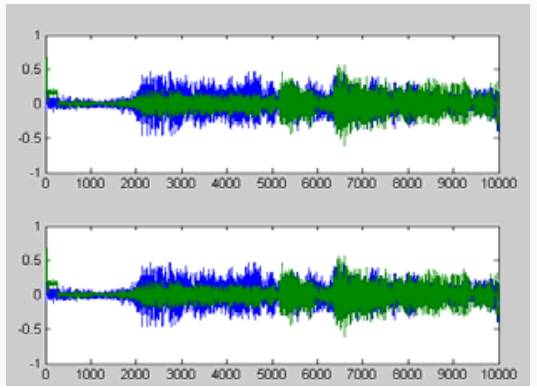

(b) Mixed signals

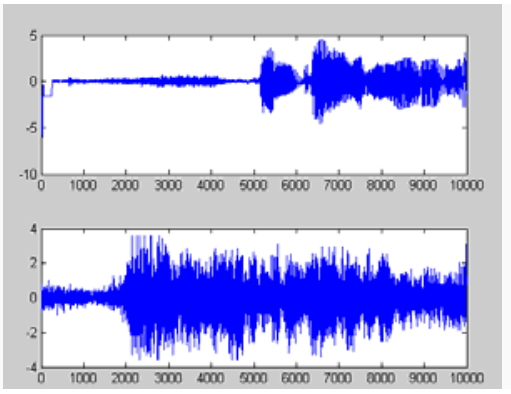

(c) IVA separation results

Figure 1 The experimental results

\section{Conclusion}

Aiming at the rapid expansion of urbanization, this paper proposed a new independent vector analysis model; it is an extension of ICA for independent multivariate speech where the sources are mixed component-wise. The article to the actual produce convolution mixture of speech signal on the rapid fixed-point IVA algorithm separation, the experiment result shows that the algorithm has a good separation actual environment of mixed speech signal and high accuracy, and the convergence speed is good. At the same time, this approximate algorithm by increasing the frequency constraints between independent greatly improve the ICA algorithm separated signal order uncertainty problem, so IVA algorithm has better adaptability, higher accuracy and great flexibility. 


\section{References}

[1] T.K im. H.T. Attias, s.-y. Lee, t.-w. Lee, Blind source separation exploiting higher-order frequency dependencies, IEEE Trans. Speech and Audio Processing, 15 (1) (2007) 70-76.

[2] E. Bingham, a. Hyva " rinen, A fast fixed-point algorithm for independent component analysis of complex-valued signals, Int. J. Neural Systems 10 (1) (2000) 1-8.

[3] Abrard F,Deville Y.A time-frequency blind signal separation method applicable to underdetermined mixtures of dependent sources. Signal Processing. 2005

[4] Y. Deville,M. Puigt,,B. Albouy.Time-frequency blind signal separation: extended methods, performance evaluation for speech sources. 2004

[5] Fadaili E M, Moreau T N,Moreau E.Non-orthogonal joint diagonalization/ zero-diagonalization for source separation based on time-frequency distributions. IEEE Transactions on Signal Processing. 2007

[6] Matthieu Puigt,Yannick Deville.Time-frequency ratio-based blind separation methods for attenuated and time-delayed source. Journal of Mechanical Systems. 2005

[7] Cédric Gouy-Pailler;Reza Sameni;Marco Congedo;Christian Jutten; Iterative Subspace Decomposition for Ocular Artifact Removal from EEG Recordings [J]. Independent Component Analysis and Signal Separation, 2009.

[8] Carlos Estêvão R. Fernandes;Pierre Comon;Gérard Favier; Blind Order Detection and Parameter Estimation of MISO-FIR Channels [J]. Independent Component Analysis and Signal Separation, 2009.

[9] Matthieu Puigt;Yannick Deville; Iterative-Shift Cluster-Based Time-Frequency BSS for Fractional-Time-Delay Mixtures [J]. Independent Component Analysis and Signal Separation, 2009.

[10] Li Rui,Li Hongwei,Wang Fasong.Dependent Component Analysis:Concepts and Main Algorithms. 2010

[11] Tirza Routtenberg,Joseph Tabrikian.MIMO-AR System Identification and Blind Source Separation for GMM-Distributed Sources. IEEE Transactions on Electromagnetic Compatibility. 2009

[12] Shi Zhenwei,Jiang Zhiguo,et al.Blind source separation with nonlinear autocorrelation and non-Gaussianity. 2009

[13] Adel Belouchrani,Karim Abedmeraim.Contrast functions for blind source separation based on time frequency distributions. . 2006

[14] Flavio Henrique Teles Vieira;Ligia Maria Carvalho Sousa;George E. Bozinis;Wesley F. Miranda;Charles Casimiro Cavalcante; Network Traffic Flow Separation and Control Through a Hybrid ICA-Fuzzy Adaptive Algorithm [J]. Independent Component Analysis and Signal Separation, 2009. 